\title{
Ganglioneuroma suprarrenal gigante: reporte de un
}

\section{caso}

\section{Giant adrenal ganglioneuroma: A case report}

\author{
Alberto Jorge Camacho-Castro, ${ }^{1}$ Iván Aguilar-Enríquez, ${ }^{1}$ Isaac Gerardo-Osuna, ${ }^{2}$ Jaime Luis Moya-Núñez ${ }^{3}$
}

\begin{abstract}
Resumen
ANTECEDENTES: Los ganglioneuromas son tumores excepcionales. Hasta el momento no existe un consenso de tratamiento, por lo que es necesario individualizar cada caso. CASO CLínICO: Paciente masculino de 36 años, con afectación intermitente de la fosa renal izquierda y el flanco ipsilateral de cuatro meses de evolución. El ultrasonido abdominal reportó una masa adyacente al riñón izquierdo, en su porción superior, de $5 \times 4 \mathrm{~cm}$, hipoecoica, sin calcificaciones. La tomografía abdominopélvica simple y contrastada evidenció una neoplasia suprarrenal izquierda, de $8 \times 5 \mathrm{~cm}$. Se programó para adrenalectomía laparoscópica izquierda. Durante el procedimiento se encontró una neoplasia suprarrenal de $9 \times 8 \mathrm{~cm}$, firmemente adherida a la vena renal ipsilateral, por lo que solo se practicó la resección parcial del tumor. La evolución del paciente fue satisfactoria y egresó al segundo día posoperatorio. El reporte de patología evidenció dos fragmentos de tejido, que en conjunto pesaron $55 \mathrm{~g}$, y por separado $5.5 \mathrm{x}$ $4.2 \mathrm{~cm}$ y $4.5 \times 2.8 \mathrm{~cm}$, respectivamente. La histología mostró un estroma constituido por células de Schwann, orientadas en dirección longitudinal y transversal, entrecruzadas en forma irregular. De acuerdo con los hallazgos histopatológicos y el estudio de inmunohistoquímica se estableció el diagnóstico de ganglioneuroma suprarrenal.

CONCLUSIONES: Los ganglioneuromas suelen provocar síntomas inespecíficos, por lo que el diagnóstico se establece de forma fortuita. Después de la resección del tumor, el pronóstico de los pacientes es excelente. Este es el primer caso de ganglioneuroma reportado en México.
\end{abstract}

PALABRAS CLAVE: Ganglioneuroma; neoplasia suprarrenal; adrenalectomía laparoscópica.

\section{Abstract}

BACKGROUND: Ganglioneuromas are exceptional tumors. At present there is no consensus on treatment and so each case must be individualized.

CLINICAL CASE: A 36-year-old man presented with an intermittent sensation of heaviness in the left renal fossa and ipsilateral flank of four-month progression. Abdominal ultrasound reported a hypoechoic mass adjacent to the left kidney at its upper portion, measuring $5 \times 4 \mathrm{~cm}$, with no calcifications. Non-contrast and contrast-enhanced abdominopelvic tomography identified an $8 \times 5 \mathrm{~cm}$ left adrenal neoplasia. The patient was programmed for laparoscopic left adrenalectomy. During the procedure, a $9 \times 8$ $\mathrm{cm}$ adrenal neoplasia was found firmly adhered to the ipsilateral renal vein, and so the tumor was only partially resected. Patient progression was satisfactory, and he was released on postoperative day two. The pathology report described two fragments of tissue, together weighing $55 \mathrm{~g}$, and separately measuring $5.5 \times 4.2 \mathrm{~cm}$ and $4.5 \times 2.8$ $\mathrm{cm}$. The histology study revealed a stroma composed of longitudinal and transversal Schwann cells that were irregularly intertwined. According to the histopathologic findings and immunohistochemical study, adrenal ganglioneuroma was diagnosed.

CONCLUSIONS: Ganglioneuromas usually cause nonspecific symptoms, resulting in incidental diagnoses. After tumor resection, patients have excellent prognosis. The case of ganglioneuroma presented herein is the first to be reported in Mexico.

KEYWORDS: Ganglioneuroma; Adrenal neoplasia; Laparoscopic adrenalectomy.
${ }^{1}$ Adscrito al servicio de Urología, Hos pital General de Culiacán Dr. Bernardo J. Gastélum, Sinaloa.

${ }^{2}$ Residente de tercer año de Urología, Hospital General de Sonora, Hermosillo. ${ }^{3}$ Patólogo.

Recibido: octubre 2017

Aceptado: julio 2018

Correspondencia

Alberto Jorge Camacho Castro ajcc7@hotmail.com

Este artículo debe citarse como Camacho-Castro AJ, Aguilar-Enríquez I, Gerardo-Osuna I, Moya-Núñez JL. Ganglioneuroma suprarrenal gigante: reporte de un caso. Rev Mex Urol. 2018 julio-agosto;78(4):298-302. DOI: https://doi.org/10.24245/revmexurol.v78i4.1772 


\section{ANTECEDENTES}

Los neuroblastomas fueron descritos originalmente por Virchow en 1863; sin embargo, al iniciar el siglo XX Zückerkandl y Kohn determinaron su origen en el tejido simpático. En 1927 se observó que un neuroblastoma puede evolucionar a ganglioneuroma. ${ }^{1}$

Los tumores neuroblásticos se originan en las células de la cresta neural del tejido nervioso simpático, incluso pueden formarse en cualquier punto del sistema paravertebral y la médula suprarrenal. La clasificación actual de este tipo de neoplasias incluye: neuroblastomas, ganglioneuroblastomas y ganglioneuromas, y los tres difieren en su grado de maduración. Los tumores inmaduros suelen ser agresivos y aparecen en pacientes más jóvenes, mientras que los maduros afectan a pacientes de mayor edad y tienden a comportarse de manera benigna. ${ }^{2}$

Los ganglioneuromas son tumores benignos que afectan frecuentemente a niños y adultos jóvenes; las mujeres son más propensas a la neoformación que los hombres. La mayor parte de estos tumores se sitúa en el retroperitoneo, su detección suele ser fortuita, porque casi siempre se mantienen hormonalmente inactivos. El examen histológico representa el único estudio capaz de diferenciar entre ganglioneuromas y neuroblastomas; generalmente se asocian con supervivencia a largo plazo libre de enfermedad, aún con tratamiento quirúrgico incompleto. Los reportes en la bibliografía de estos tumores son pocos debido a su rareza.,

\section{CASO CLÍNICO}

Paciente masculino de 36 años de edad, sin antecedentes de importancia para el padecimiento actual, con una sensación intermitente de pesantez en la fosa renal izquierda y el flanco ipsilateral, de cuatro meses de evolución, sin síntomas agregados. Después de su recepción en el servicio médico se efectuó un ultrasonido abdominal que reportó una masa adyacente en el riñón izquierdo, en su porción superior, de aproximadamente $5 \times 4 \mathrm{~cm}$, hipoecoica, sin calcificaciones en su interior. La tomografía computada abdominopélvica simple y contrastada evidenció una neoplasia suprarrenal izquierda, de aproximadamente $8 \times 5 \mathrm{~cm}$, con 22 Unidades Hounsfield en promedio, sin reforzamiento significativo después de la aplicación del medio de contraste (Figura 1). Con estos hallazgos se envió al servicio de Urología del Hospital General de Culiacán Dr. Bernardo J. Gastélum.

A la exploración física se observó al paciente con buen estado general, sin percepción de masa abdominal a la palpación. La determinación de

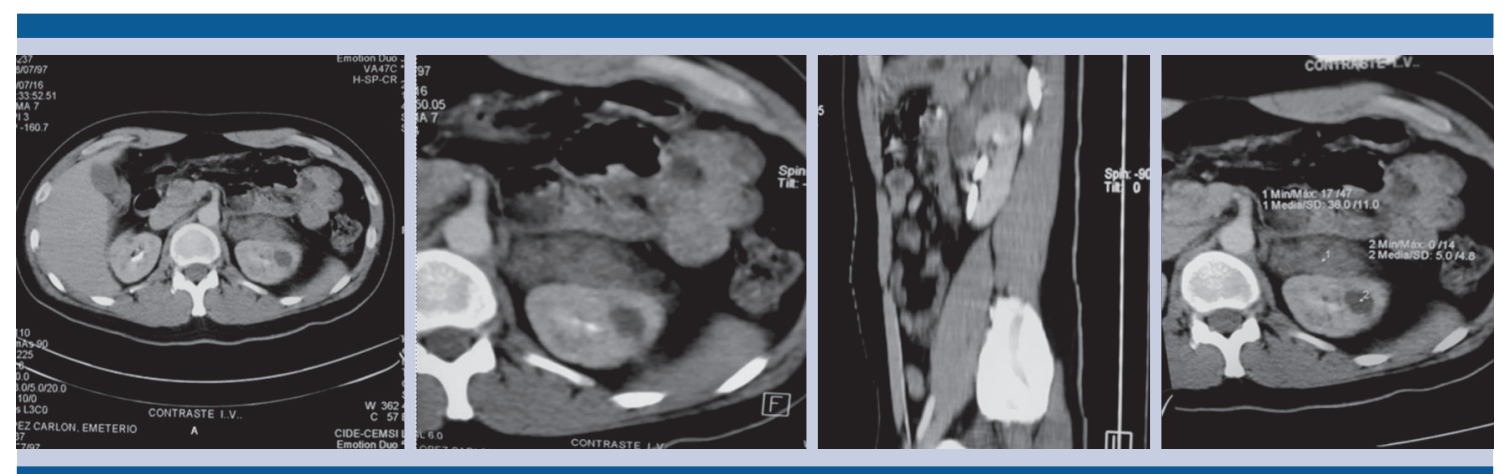

Figura 1. Tomografía abdominopélvica simple y contrastada. 
metanefrinas reportó concentraciones dentro de los parámetros normales (metanefrina 65 pg/ $\mathrm{mL}$, normetanefrina $110 \mathrm{pg} / \mathrm{mL}$ y metanefrinas totales $175 \mathrm{pg} / \mathrm{mL}$ ). El gammagrama de MIBG resultó negativo y los estudios de laboratorio preoperatorios se encontraron sin alteraciones.

Se programó al paciente para adrenalectomía laparoscópica izquierda. Durante el procedimiento se encontró una neoplasia suprarrenal de $9 \times 8 \mathrm{~cm}$, firmemente adherencia a la vena renal ipsilateral, por lo que solo se resecó el tumor parcialmente. El procedimiento tuvo una duración aproximada de 2 h y 30 minutos, con sangrado estimado de 150 cc.
La evolución del paciente fue satisfactoria, con tolerancia a la vía oral y deambulación al día siguiente de la operación. Egresó al segundo día posoperatorio y se retiró el drenaje al quinto día del procedimiento.

El reporte de patología evidenció dos fragmentos de tejido, que en conjunto pesaron $55 \mathrm{~g}$ y por separado midieron $5.5 \times 4.2$ y $4.5 \times 2.8 \mathrm{~cm}$, respectivamente; los cortes histológicos mostraron un estroma constituido por células de Schwann, orientadas en dirección longitudinal y transversal, entrecruzadas en forma irregular; se identificaron con facilidad filetes nerviosos y células ganglionares maduras (Figura 2), con lo que se estableció el diagnóstico histopatológico

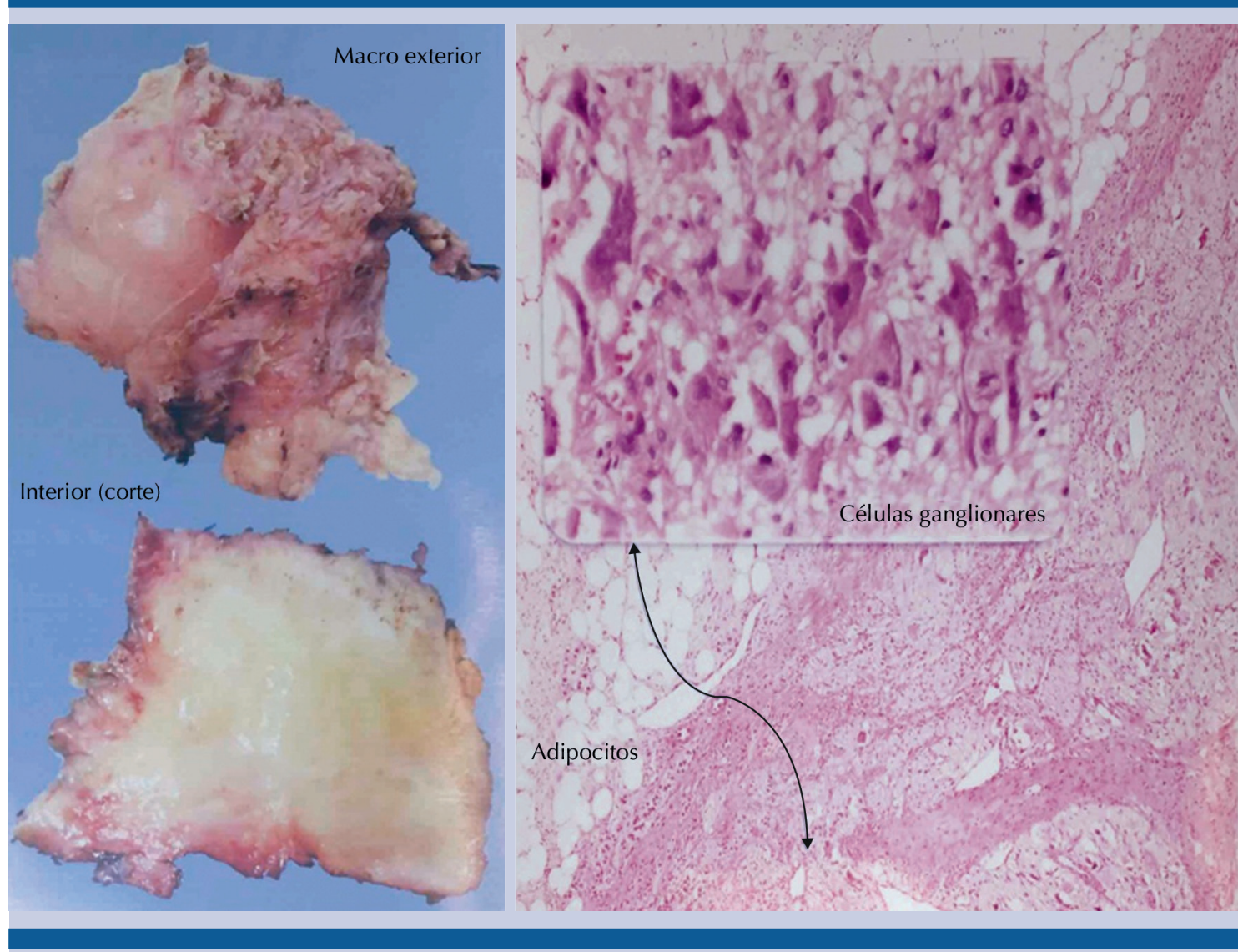

Figura 2. Corte macroscópico y microscópico del tumor. 
de ganglioneuroma suprarrenal. Debido a la rareza del caso y la identificación de tumores compuestos se realizó el estudio de inmunohistoquímica (Figura 3), que reportó positividad para proteína S-100 (3+), CD-56 (3+) y cromogranina (3+); por tanto, se comprobó el diagnóstico de ganglioneuroma.

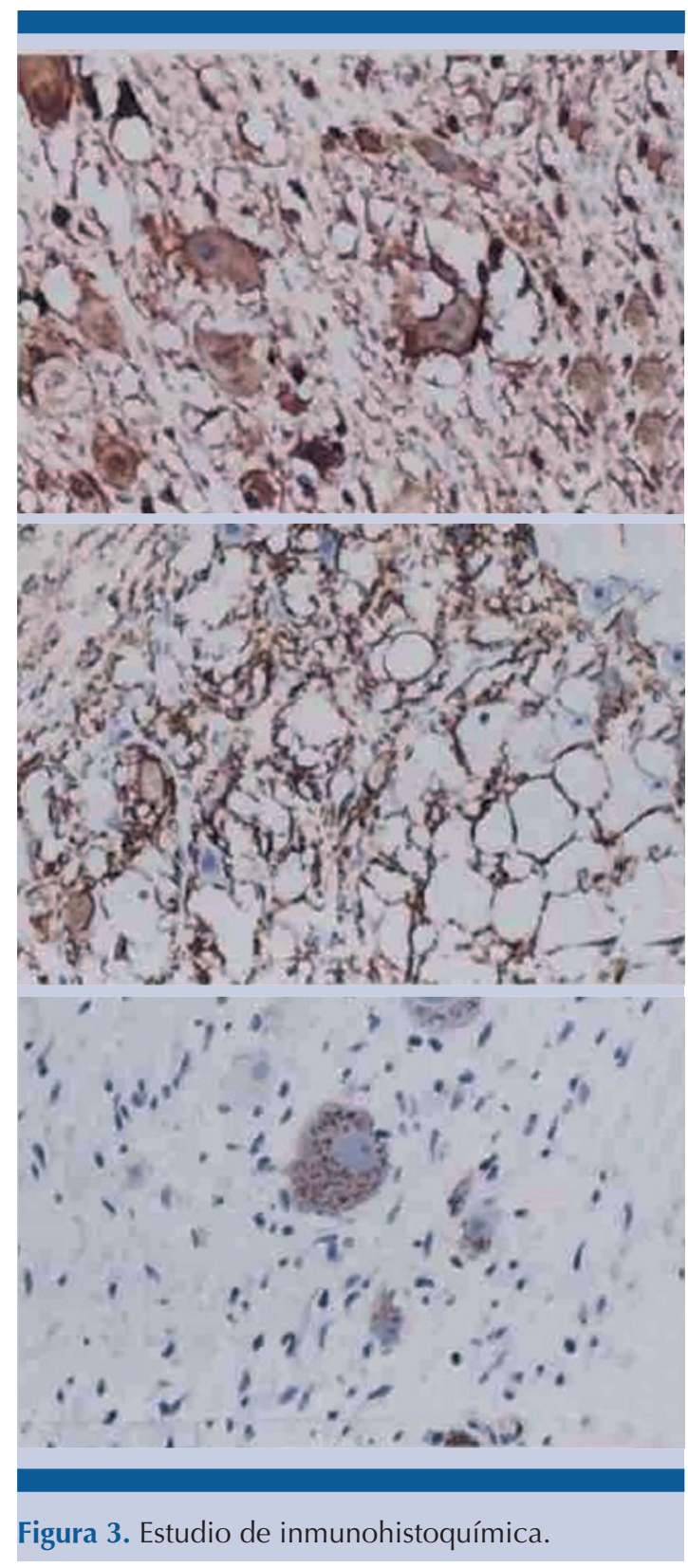

\section{DISCUSIÓN}

Del grupo de tumores neuroblásticos, los ganglioneuromas son los más raros y benignos. El retroperitoneo, mediastino y glándulas suprarrenales son los sitios más comunes de afectación; sin embargo, pueden encontrarse en el cuello o la pelvis, incluso en el conducto gastrointestinal. Los ganglioneuromas están compuestos por células de Schwann maduras, células ganglionares y fibras nerviosas; los estudios de laboratorio no muestran atipia celular, actividad mitótica ni necrosis. En casos excepcionales se han identificados tumores compuestos, con elementos de feocromocitoma, ganglioneuroblastoma y neuroblastoma, que pueden evolucionar a un tumor maligno. ${ }^{5}$

El ultrasonido, la tomografía computada y resonancia magnética reportan una masa bien circunscrita, oval o lobulada. El ultrasonido suele evidenciar una masa homogénea e hipoecoica; la tomografía sin contraste muestra los ganglioneuromas con baja atenuación y homogéneos, incluso en $20 \%$ de los casos puede observarse una calcificación fina y moteada, estos tienden a rodear o desplazar los grandes vasos, sin ocasionar compresión u oclusión; además, puede visualizarse refuerzo heterogéneo retardado con el medio de contraste, debido a la coexistencia de matrices mixoides en el tumor., ${ }^{6,7}$ En el paciente de este estudio, la neoplasia no mostró refuerzo significativo con la aplicación del medio de contraste, solo se evidenció una firme adherencia, con desplazamiento de la vena renal sin comprimir su luz.

La resonancia magnética muestra baja intensidad en la señal T1 y alta intensidad heterogénea T2, esta última depende de la combinación del material mixoide y las células ganglionares $y$, al igual que en la tomografía, se encuentra un retraso en el reforzamiento. ${ }^{9}$ El $57 \%$ de los 
casos se reportan con captación del marcador meta-yodo-bencil-guanidina y la mayor parte de los tumores producen cantidades elevadas de catecolaminas.

La adrenalectomía laparoscópica es el tratamiento de elección en pacientes con neoplasias adrenales, sobre todo en tumores menores de $5 \mathrm{~cm}$. Por tratarse de un método de mínima invasión, el acceso elegido para este paciente permitió mejor recuperación y se logró la extirpación de la neoplasia casi en su totalidad, pues fue imposible la remoción del segmento adherido a la vena renal. ${ }^{10}$

\section{CONCLUSIONES}

Los ganglioneuromas son tumores excepcionales. Hasta el momento no existe concenso de tratamiento, por lo que es necesario individualizar cada caso. Puesto que los síntomas son inespecíficos, el diagnóstico se establece de forma fortuita. Afortunadamente, el pronóstico de la neoplasia, posterior a su resección, es excelente. Este es el primer caso de ganglioneuroma reportado en México.

\section{REFERENCIAS}

1. Zhou $Y$, Liang $Q$, Wen-Ting $\mathrm{O}$, et al. Laparoscopic resection of primary adrenal ganglionueroma: A case report and review of the literature. Oncol Let 2015;1-4.

2. Koktener A, Koschan D, Akin K, et al. Incidentally found retroperitoneal ganglioneuroma in an adult. Indian J Surg 2015;77(Suppl 1):S3-S5.

3. Chen $P$, Lin $C$, Jin L, et al. A case report of giant adrenal ganglioneuroma. Urology Case Reports 2017;13:37-38.

4. Tobías-Machado M, Rincón F, Lasmar M, et al. Suprarrenalectomía laparoscópica retroperitoneal como opción mínimamente invasiva en el tratamiento de tumores adrenales. Arch Esp Urol 2006;59(1):49-54.

5. Mohammed M, Elmouhadi S., Elaloui $M$, et al. Imaging features of adrenal ganglioneuroma: a case report. BMC 2014;7:791.

6. Méndez-Pérez V, Palmerín-Bucio M. Ganglioneuroma mediastinal en un adulto. Informe de un caso y revisión de la literatura. Gac Med Mex. 2010;146(3):216-218.

7. Guevara J, Cruz H. Ganglioneuroma de la región cervical C1-C3: informe de un caso. Cir Cir 1997;65:18-20.

8. Cooper SH, Baldwin R, Mlynarczyk C, et al. Renal ganglioneuroma presenting as an apparent renal artery aneurism. Urol C Rep 2015;3:86-88.

9. Li L, Shao J, Gu j, et al. Adrenal ganglioneuromas: experience from a retrospective study in a chinese population. Urol 2014;11(2).

10. Torregrosa L, Granada J, Cervera S. Ganglioneuroma retroperitoneal en un hospital universitario: reporte de caso. Univ Med 2013;54(3):396-400.

11. Celik B, Unal G, Ozgultekin R, et al. Adrenal gaglioneuroma. Br J Sur 1996;83:263. 